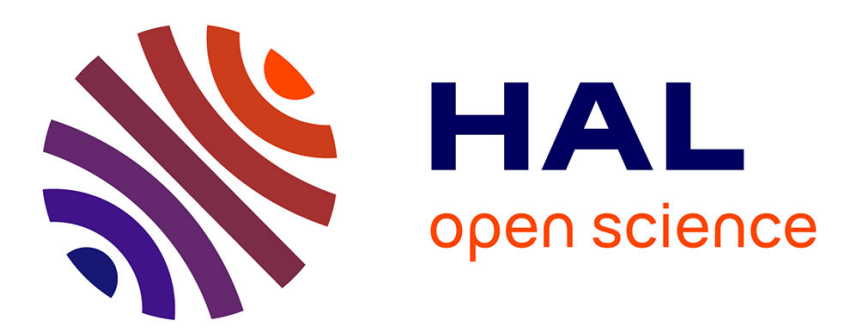

\title{
Input and State Observability of Network Systems with Time-Varying Topology
}

\author{
Sebin Gracy, Federica Garin, Alain Kibangou
}

\section{To cite this version:}

Sebin Gracy, Federica Garin, Alain Kibangou. Input and State Observability of Network Systems with Time-Varying Topology. IEEE Transactions on Control of Network Systems, 2019, 6 (2), pp.897-905. 10.1109/TCNS.2018.2880304 . hal-01918497

\section{HAL Id: hal-01918497 https://hal.science/hal-01918497}

Submitted on 11 Nov 2018

HAL is a multi-disciplinary open access archive for the deposit and dissemination of scientific research documents, whether they are published or not. The documents may come from teaching and research institutions in France or abroad, or from public or private research centers.
L'archive ouverte pluridisciplinaire HAL, est destinée au dépôt et à la diffusion de documents scientifiques de niveau recherche, publiés ou non, émanant des établissements d'enseignement et de recherche français ou étrangers, des laboratoires publics ou privés. 


\title{
Input and State Observability of Network Systems with Time-Varying Topology
}

\author{
Sebin Gracy, Federica Garin and Alain Y. Kibangou.
}

\begin{abstract}
We aim to study the problem of reconstructing the initial state as well as the sequence of unknown inputs (ISO) for linear network systems having time-varying topology. Evolution of such systems can be represented by a collection of graphs $\left\{\mathcal{G}_{k}\right\}$. We find conditions under which the system with a pattern of fixed zeros imposed by $\left\{\mathcal{G}_{k}\right\}$ is ISO: a) for almost all choices of edge weights in $\left\{\mathcal{G}_{k}\right\}$ (structural ISO); and b) for all nonzero choices of edge weights in $\left\{\mathcal{G}_{k}\right\}$ (strongly structural ISO). We introduce two suitable descriptions of the whole collection of graphs $\left\{\mathcal{G}_{k}\right\}$ named as dynamic graph and dynamic bipartite graph. Two equivalent characterizations of structural ISO are then stated in terms of existence of a linking and a matching of suitable size in the dynamic graph and in the dynamic bipartite graph, respectively. For strongly structural ISO, we provide a sufficient condition and a necessary condition, both concerning the existence of a uniquely restricted matching of suitable size in the dynamic bipartite graph and in a subgraph of it. When there is no direct feedthrough of the input on the measurements, the two conditions can be merged to give rise to a necessary and sufficient condition.
\end{abstract}

Index Terms - Linear Network systems, Time-varying topology, Input and State Observability (ISO), Structural ISO, Strongly Structural ISO, Cyber-Physical Security.

\section{INTRODUCTION}

The idea of achieving system-theoretic properties like controllability and observability in network systems has recently drawn the attention of control theorists. While there exist algebraic characterizations of such properties, these depend on exact knowledge of coefficients of system matrices, which are often not available in network systems. Moreover, for large networks, checking such characterizations is computationally heavy. This prompts one to use graphs to represent and study such systems, by taking recourse to the notion of structured systems. In such systems, the system matrices have fixed zero patterns. The positions that are not fixed to zero are referred to as free parameters. Under such a setting, on the one hand, one seeks structural results i.e., results that are true for almost all choices. On the other hand, there exists yet another line of work where one wants to ensure that results are true for all non-zero choice of free parameters, referred to as strongly structural (s-structural) results.

The existing literature on structured systems is focused on time-invariant topologies, and is primarily concerned with controllability and observability (see seminal papers [1],

The authors are with Univ. Grenoble Alpes, CNRS, Inria, Grenoble INP, GIPSA-Lab, F-38000, Grenoble, France. (e-mail: sebin.gracy@inria.fr, federica.garin@inria.fr, alain.kibangou@univ-grenoble-alpes.fr)
[2], [3]; also see, surveys, [4], [5]). However, in reality, one can find networks that exhibit time-varying behavior. For instance, in social networks, the individuals are in contact with each other only for a finite time interval, and such relationships are often intermittent and recurrent [6], whereas in communication networks, bursty transmission, packet loss, variation of channel parameters etc., results in intermittent communication [7] which leads to changes in the topology of such networks. This motivates us to turn our attention to time-varying topologies.

Dynamical systems could be affected by the presence of unknown inputs, which could encompass unmodeled parts of a system, faults or malicious attacks [8]. There exist scenarios wherein it is essential to reconstruct not only the state vector of a network but also the unknown inputs affecting it, as was pointed out, among others, in $[9,10]$. The problem of reconstructing both the initial state and the unknown input is referred to as input and state observability (ISO).

The problem of structural ISO for linear time-invariant (LTI) systems has been studied in [11], [12]. Recently, under the assumption that the topology of the network remains fixed but the weights along the edges vary with time, a characterization for structural ISO of linear time-varying (LTV) network systems accounting for the presence of multiple unknown inputs was given by the authors in [13]. On the other hand, under suitable assumptions, a characterization for s-structural ISO for LTI network systems affected by a single unknown input was first given in [14], which, in [13], was extended for LTV network systems while also accounting for multiple unknown inputs. The findings in [13] had certain limitations. First, the following assumptions were made on the structure of input and output matrices: each unknown input affected exactly one state; each state was affected by at most one unknown input; direct measurements of a few states were available (so-called dedicated sensors); and zero feedthrough. As a consequence of such assumptions, the problem of ISO was equivalent to an observability problem of a suitably defined subsystem. Thereafter, one took advantage of this equivalence to study structural (resp. s-structural) ISO. However, the extension of these results accounting for arbitrary structure of input and output matrices is non-trivial, since one cannot rephrase ISO as an equivalent problem in observability. Second, these results do not extend immediately to the case wherein the topology of the network varies over an interval. To the best of our knowledge, for linear network systems wherein the underlying topology varies over an 
interval, a characterization for structural (resp. s-structural) ISO, factoring in arbitrary time-varying input, output and feedthrough matrices, is still missing. The aim of the present paper is to close this gap.

Our main contributions are threefold. Firstly, for non-zero feedthrough, we provide an algebraic characterization for ISO of linear network systems (see Prop. 2), which also applies, in particular, to zero feedthrough. Secondly, for the case where the topology of the network evolves over an interval, we give two graphical characterizations for structural ISO that also accounts for non-zero feedthrough (see Theorem 1). Thirdly, under the aforesaid setting, for s-structural ISO, we provide two conditions which are sufficient (item 1 in Theorem 2) and necessary (item 2 in Theorem 2). Furthermore, under some assumptions on the feedthrough matrix, the two conditions can be combined to give a necessary and sufficient condition for s-structural ISO (see Corollary 1). The results, namely Theorem 1 and Theorem 2, are also applicable to timeinvariant topologies, and hence generalize the results in [15] (resp. [16]) to the case of structural (resp. s-structural) ISO, and also those in [13] by accounting for arbitrary structures of input, output and feedthrough matrices.

The organization of the present paper is as follows: We first state the problem under consideration in Section II. Thereafter, in Section III, we give an algebraic characterization of ISO for LTV systems with direct feedthrough matrix. Section IV lays the groundwork for migrating to a graphical characterization. Section $\mathrm{V}$ gives the first main result that deals with structural ISO, while Section VI introduces the second main result that concerns s-structural ISO. Section VII gives some concluding remarks.

\section{Notations}

$\mathbb{R}$ and $\mathbb{Z}$ denote the set of real numbers and integers, respectively. $I_{N}$ indicates an identity matrix of size $N$. $A=\operatorname{diag}\left(A_{1}, A_{2}, \ldots, A_{N}\right)$ represents a block diagonal matrix whose blocks along the diagonal are $A_{1}, A_{2}, \ldots, A_{N}$. $\left\{A_{k}\right\}_{k_{0}}^{k_{1}}$ denotes a sequence of matrices $A_{k}$ with $k=k_{0}, k_{0}+$ $1, \cdots, k_{1} \cdot|\mathcal{X}|$ indicates cardinality of a set $\mathcal{X} \cdot \operatorname{rank}(A)$ denotes the rank of a matrix $A$. $\left[k_{0}, k_{1}\right]$ represents the discrete interval $k_{0}, k_{0}+1, \ldots, k_{1}-1, k_{1}$.

\section{PRoblem Formulation}

Consider a discrete-time linear network of $N$ nodes whose dynamics are influenced by themselves and also by a set of $P$ external unknown inputs, and whose measurements are taken from a set of $M$ nodes. These nodes are referred to as state, input and output nodes, respectively, and the corresponding sets, are respectively denoted by $X, U$ and $Y$. The graph $\mathcal{G}_{k}=\left(\mathcal{V}, \mathcal{E}_{k}\right)$, where $\mathcal{V}=X \cup U \cup Y, \mathcal{E}_{k}=\mathcal{E}_{A_{k}} \cup \mathcal{E}_{B_{k}} \cup$ $\mathcal{E}_{C_{k}} \cup \mathcal{E}_{D_{k}}$, represents the underlying system at time instant $k$. $\mathcal{E}_{A_{k}} \subseteq X \times X$ (resp. $\mathcal{E}_{B_{k}} \subseteq U \times X$ ) represents the edges going from a state (resp. input) vertex to another state vertex. $\mathcal{E}_{C_{k}} \subseteq X \times Y$ (resp. $\mathcal{E}_{D_{k}} \subseteq U \times Y$ ) represents the edges going from a state (resp. input) vertex to an output vertex. We define $w_{k}: \mathcal{E}_{k} \rightarrow \mathbb{R}$, a function mapping the edges to their weights.
The dynamics of such a linear network system are given as follows:

$$
\begin{aligned}
x_{k+1} & =A_{k} x_{k}+B_{k} u_{k} \\
y_{k} & =C_{k} x_{k}+D_{k} u_{k}
\end{aligned}
$$

with state vector $x_{k} \in \mathbb{R}^{N}$, unknown input vector $u_{k} \in \mathbb{R}^{P}$ and output vector $y_{k} \in \mathbb{R}^{M}$. Notice that each node has firstorder dynamics. The matrix $A_{k}$ has entries equal to the edge weights between the state vertices at time $k$. Hence, it satisfies the following condition:

$$
\left[A_{k}\right]_{i j}= \begin{cases}w_{k}\left(x_{j}, x_{i}\right) & \text { if }\left(x_{j}, x_{i}\right) \in \mathcal{E}_{A_{k}} \\ 0 & \text { otherwise }\end{cases}
$$

Analogous conditions are satisfied by the matrices $B_{k}, C_{k}$ and $D_{k}$. Throughout this paper a numerical choice of free parameters is regarded as a choice of edge weights, and hence the terms "free parameters" and "edge weights" are used interchangeably, depending on the context.

From hereon, over a given interval $\left[k_{0}, k_{1}\right]$, the collection of corresponding (possibly) time-varying graphs is denoted with $\left\{\mathcal{G}_{k}\right\}$. The term time-varying should be understood in the following sense: any two graphs belonging to $\left\{\mathcal{G}_{k}\right\}$ have the same vertex sets, but might have different edge sets.

We assume that, in general, for any $k_{0}, k_{0}+1$ the graphs $\mathcal{G}_{k_{0}}=\left(\mathcal{V}, \mathcal{E}_{k_{0}}\right)$ and $\mathcal{G}_{k_{0}+1}=\left(\mathcal{V}, \mathcal{E}_{k_{0}+1}\right)$ might be different i.e., $\mathcal{E}_{k_{0}} \neq \mathcal{E}_{k_{0}+1}$. For an illustration see Figures 1a, 1b wherein $\mathcal{G}_{k}$ is periodic with periodicity 2 , i.e., $\mathcal{G}_{k}$ alternates between the depicted topologies with period 2 .

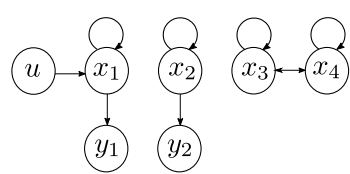

(a) $\mathcal{G}_{2 k}$

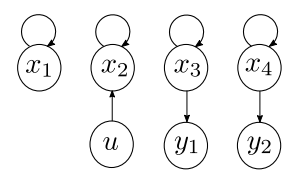

(b) $\mathcal{G}_{2 k+1}$
Fig. 1: Evolution of an LTV system represented by timevarying periodic graphs with periodicity 2 .

We assume that the sequence of (possibly) time-varying topologies is known. The setup described herein differs from the one in [17] in the sense that there is no switching rule governing the change in the network topology at each time instant. Furthermore, it also differs from the framework in [9] in the sense that while the unknown inputs are free to influence any nodes at each time-instant, they have no impact on the evolution of the topology of the network.

In this paper, we study network systems with time-varying topologies. Firstly, we find algebraic conditions for the system to be ISO. Secondly, we find graphical conditions under which i) the system is structurally ISO (see Sect. V), and ii) the system is s-structurally ISO (see Sect. VI).

\section{Algebraic Characterizations}

The objective of this section is to provide two algebraic criteria for ISO, which is defined as follows.

Definition 1: System (1) is ISO over $\left[k_{0}, k_{1}\right]$ if the initial condition $x_{k_{0}} \in \mathbb{R}^{N}$ and the unknown inputs sequence 
$\left\{u_{k_{0}}, u_{k_{0}+1}, \ldots, u_{k_{1}-1}\right\}$ can be uniquely recovered from the measurements $\left\{y_{k_{0}}, y_{k_{0}+1}, \ldots, y_{k_{1}}\right\}$.

Let $\Theta_{k_{0}, k_{1}}, \Gamma_{k_{0}, k_{1}}$ and $\Psi_{k_{0}, k_{1}}$ represent the observability matrix, invertibility matrix and input and state observability (ISO) matrix, respectively, over the interval $\left[k_{0}, k_{1}\right]$. These are defined as follows:

$$
\begin{aligned}
& \Theta_{k_{0}, k_{1}}=\left[\begin{array}{c}
{ }^{C_{k_{0}}} \\
{ }_{{ }_{k_{0}}+1} A_{k_{0}} \\
{ }_{k_{0}+2}{ }^{A_{k_{0}}+1} A_{k_{0}} \\
\vdots \\
{ }_{C_{k_{1}} A_{k_{1}-1} \cdots A_{k_{0}}}
\end{array}\right], \\
& \Gamma_{k_{0}, k_{1}}=\left[\begin{array}{cccc}
D_{k_{0}} & \cdots & \cdots & 0 \\
C_{k_{0}+1 B_{k_{0}}} & D_{k_{0}+1} & \cdots & 0 \\
\vdots & \cdots & \cdots & \vdots \\
C_{k_{1}} A_{k_{1}-1} \cdots A_{k_{0}+1} B_{k_{0}} & \cdots & C_{k_{1} B_{k_{1}-1}} & D_{k_{1}}
\end{array}\right], \\
& \Psi_{k_{0}, k_{1}}=\left[\Theta_{k_{0}, k_{1}} \Gamma_{k_{0}, k_{1}}\right] .
\end{aligned}
$$

Using the notation $u_{k_{0}: k_{1}}=\left(u_{k_{0}}^{T}, u_{k_{0}+1}^{T}, \ldots, u_{k_{1}}^{T}\right)^{T}$ and $y_{k_{0}: k_{1}}=\left(y_{k_{0}}^{T}, y_{k_{0}+1}^{T}, \ldots, y_{k_{1}}^{T}\right)^{T}$, it is immediate to obtain $y_{k_{0}: k_{1}}=\Theta_{k_{0}, k_{1}} x_{k_{0}}+\Gamma_{k_{0}, k_{1}} u_{k_{0}: k_{1}}=\Psi_{k_{0}, k_{1}}\left[\begin{array}{c}x_{k_{0}} \\ u_{k_{0}: k_{1}}\end{array}\right]$. Then the following result is immediate (see [18] for a similar result).

Proposition 1: System (1) is ISO over $\left[k_{0}, k_{1}\right]$ if and only if $\operatorname{rank}\left(\Psi_{k_{0}, k_{1}}\right)=N+\left(k_{1}-k_{0}\right) P+\operatorname{rank}\left(D_{k_{1}}\right)$.

Notice that Prop. 1 characterizes ISO in terms of products of the system matrices. Consequently, the corresponding zero pattern is lost. Hence, in this subsection, we will focus on providing an alternative characterization that preserves the zero pattern of the system matrices. We define

$$
\mathcal{J}_{k_{0}, k_{1}}=\left[\begin{array}{ccc}
\mathbf{D}_{k_{0}, k_{1}-1} & 0_{\left(k_{1}-k_{0}\right) M \times P} & \\
0_{M \times\left(k_{1}-k_{0}\right) P} & D_{k_{1}} & \mathcal{Q}_{k_{0}, k_{1}} \\
\mathbf{B}_{k_{0}, k_{1}-1} & 0_{\left(k_{1}-k_{0}\right) N \times P} &
\end{array}\right]
$$

where $\quad \mathbf{D}_{k_{0}, k_{1}-1}=\operatorname{diag}\left(D_{k_{0}}, D_{k_{0}+1} \ldots D_{k_{1}-1}\right)$, $\mathbf{B}_{k_{0}, k_{1}-1}=\operatorname{diag}\left(B_{k_{0}}, B_{k_{0}+1} \ldots B_{k_{1}-1}\right)$,

$$
\mathcal{Q}_{k_{0}, k_{1}}=\left[\begin{array}{ccccc}
C_{k_{0}} & 0 & \ldots & \ldots & 0 \\
0 & C_{k_{0}+1} & \cdots & \cdots & 0 \\
\vdots & \cdots & \cdots & \cdots & \vdots \\
0 & \cdots & \cdots & \cdots & C_{k_{1}} \\
A_{k_{0}} & -I_{N} & \cdots & \cdots & 0 \\
0 & A_{k_{0}+1} & -I_{N} & \cdots & 0 \\
\cdot & \cdots & \cdots & \cdots & \vdots \\
0 & \cdots & \cdots & A_{k_{1}-1} & -\dot{I}_{N}
\end{array}\right] .
$$

Notice that $\mathcal{J}_{k_{0}, k_{1}}$ has $\left(\left(k_{1}-k_{0}+1\right) M+\left(k_{1}-k_{0}\right) N\right)$ rows and $\left(k_{1}-k_{0}+1\right) P+\left(k_{1}-k_{0}+1\right) N$ columns. Also, both the state equation and output equation at each time instant can be expressed as a linear combination of $x_{k_{0}}, x_{k_{0}+1}, \ldots, x_{k_{1}}$ as well as $u_{k_{0}}, u_{k_{0}+1}, \ldots, u_{k_{1}}$. A suitable column permutation of $\mathcal{J}_{k_{0}, k_{1}}$ (denoted as $\tilde{\mathcal{J}}_{k_{0}, k_{1}}$ ) yields

$$
\tilde{\mathcal{J}}_{k_{0}, k_{1}}\left[\begin{array}{c}
u_{k_{0}: k_{1}-1} \\
x_{k_{0}: k_{1}} \\
u_{k_{1}}
\end{array}\right]=\left[\begin{array}{c}
y_{k_{0}: k_{1}} \\
\mathbf{0}_{\left(k_{1}-k_{0}\right) N}
\end{array}\right] .
$$

Therefore, the following result is immediate.

Proposition 2: System (1) is ISO over $\left[k_{0}, k_{1}\right]$ if and only if $\operatorname{rank}\left(\mathcal{J}_{k_{0}, k_{1}}\right)=\left(k_{1}-k_{0}\right) P+\left(k_{1}-k_{0}+1\right) N+\operatorname{rank}\left(D_{k_{1}}\right)$.

In the sequel, we seek a graphical characterization for ISO.

\section{TRANSITION TO A GRAPHICAL CHARACTERIZATION}

The focus of this section is to develop the framework for migrating to a graphical characterization of ISO.

\section{A. Structured Matrices}

Since the ISO matrix $\Psi_{k_{0}, k_{1}}$ contains products of the system matrices, the coefficients of $\Psi_{k_{0}, k_{1}}$ are either fixed zeros or polynomials in the free parameters of the system matrices. Inspired from [4], consider a matrix $A$ that has fixed zero positions and all its other entries are non-zero polynomials in free parameters (say $\lambda_{1}, \lambda_{2}, \ldots, \lambda_{\mu}$ ). Let us call such a matrix as a structured matrix. Let $\lambda=\left[\begin{array}{llll}\lambda_{1} & \lambda_{2} & \ldots & \lambda_{\mu}\end{array}\right]$ denote the vector of free parameters in $\mathbb{R}^{\mu}$. Each vector in $\mathbb{R}^{\mu}$ denotes a particular numerical choice of free parameters. We use the term pattern matrix to represent a particular instance of a structured matrix, and we define it as follows.

Definition 2: Let $A$ be a structured matrix. $A$ is a pattern matrix if each non-zero polynomial in $A$ is of the form $\lambda_{i}$ with all $i$ 's being distinct.

\section{B. Relevant Graph Terminology}

The zero/non-zero pattern of structured matrices allows one to study structured systems by employing tools from graph theory, and hence we will briefly recall a few graph-theoretic notions. Given a graph $\mathcal{G}=(\mathcal{V}, \mathcal{E})$ we say that two paths are vertex-disjoint if they do not have any vertex in common. Next, we define a linking in a graph:

Definition 3: Let $S$ and $T$ be two sets of vertices of a directed graph. A collection of vertex-disjoint paths from set $S$ to set $T$ is called a linking from $S$ to $T$.

We say that a linking saturates a vertex if the said vertex is one of the vertices along the paths in the linking.

The notion of matching, which is closely related to linking, is defined as follows:

Definition 4: A matching is a collection of edges such that no two edges share a vertex.

The size of a matching is the number of edges contained in it; if a matching has maximum size among all the matchings in the same graph, then it is a maximum matching. We say that a matching saturates a vertex if the said vertex is one of the vertices of the edges contained in the matching. Closely related is the concept of uniquely restricted matching, which is given by the following definition.

Definition 5 (Definition 2.4 [19]): Let $\mathcal{B}=\left\{V^{+}, V^{-}, E\right\}$ be a bipartite graph. A matching of size $t$ is said to be uniquely restricted if it is the only matching of size $t$ in $\mathcal{B}$ between $\left\{i_{1}, \ldots, i_{t}\right\}$ and $\left\{j_{1}, \ldots, j_{t}\right\}$, where $\left\{i_{1}, \ldots, i_{t}\right\} \in V^{+}$and $\left\{j_{1}, \ldots, j_{t}\right\} \in V^{-}$.

\section{Ranks of structured matrices}

The rank of a structured matrix $A$ is evaluated with respect to a choice of free parameters. However, notions like term-rank and generic rank do not depend on the choice of free parameters, and hence in the following we shall briefly discuss the same. We shall do so by recalling relevant material from Chapter 2 in [4]. 
We can associate a bipartite graph to the structured matrix $A$ in the following manner; $\mathcal{B}(A)=\left\{V^{+}, V^{-}, \mathcal{E}(A)\right\}$ where $V^{+}$is the set of all columns in $A, V^{-}$is the set of all rows in $A$ and $\mathcal{E}(A)=\left\{(i, j) \mid i \in V^{+}, j \in V^{-},[A]_{j i} \neq 0\right\}$ is the edge set corresponding to positions in $A$ being non-zero polynomials in the free parameters. With the bipartite graph $\mathcal{B}(A)$ in place, the definition of term-rank $(A)$ follows.

Definition 6: The term-rank $(A)$ is equal to the maximum size of a matching in the bipartite graph $\mathcal{B}(A)$.

As far as the notion of generic rank of a structured matrix is concerned, recall that the entries of $\mathrm{A}$ are polynomials in $\mu$ free parameters. We know that any subdeterminant of $A$ is a polynomial in the free parameters $\lambda_{1}, \lambda_{2}, \ldots, \lambda_{\mu}$, which brings us to the following definition.

Definition 7: The generic rank (denoted as gen-rank) of $A$ is the maximum size of a square submatrix whose determinant is a non-zero polynomial.

It turns out that the $\operatorname{rank}(A)$ is the same for almost all choices of free parameters of $A$. This can be immediately seen by noticing that the aforementioned polynomial yields zero only when evaluated for elements in its zero set.

The main results from [4] that would be used in the sequel are given in the following result.

Lemma 1 (Chapter 2 [4]): Let $A$ be a structured matrix having $\lambda \in \mathbb{R}^{\mu}$ as its vector of free parameters. Then the following statements are true:

1) gen-rank $(A)=\max _{\lambda \in \mathbb{R}^{\mu}} \operatorname{rank}(A)$;

2) For almost all choices of $\lambda, \operatorname{rank}(A)=\operatorname{gen}-\operatorname{rank}(A)$;

3) gen-rank $(A)$ is bounded from above by term-rank $(A)$; If $A$ is a pattern matrix, gen-rank $(A)=\operatorname{term}-\operatorname{rank}(A)$

Next, we recall a result which gives a necessary and sufficient condition such that a pattern matrix has the same rank for every non-zero choice of free parameters.

Lemma 2 (Thm. 3.9 [19]): Let $A$ be an $m \times n$ pattern matrix and $\mathcal{B}(A)$ be a bipartite graph obtained from $A$, and let $r$ be a nonnegative integer. Then the following are equivalent:

1) $\operatorname{rank}(A)$ equals $r$ for every non-zero choice of free parameters.

2) In $\mathcal{B}(A)$, there exists no matching of size greater than $r$, and there exists at least one uniquely restricted matching of size $r$.

\section{Dynamic Graph}

Inspired by [4], we introduce the notion of dynamic graph, denoted as $\mathcal{S}_{k_{0}, k_{1}}$, and we show a relation between the entries in $\Psi_{k_{0}, k_{1}}$ and the paths in $\mathcal{S}_{k_{0}, k_{1}}$.

The dynamic graph is constructed in the following manner: First, create $\left(k_{1}-k_{0}+1\right)$ copies of vertex sets $X, U$ and $Y$, respectively. Label these copies as $X_{k_{0}}, X_{k_{0}+1}, \ldots, X_{k_{1}}$. Analogously label the copies of $U$ and $Y$. Let $x_{k}^{i}$ (resp. $u_{k}^{i}$ and $\left.y_{k}^{i}\right)$ be the vertex associated with the $i$ th entry of the vertex set $X_{k}$ (resp. $U_{k}$ and $Y_{k}$ ). Second, we assign edges and their weights as follows: There exists an edge between vertices $x_{k}^{i}$ and $x_{k+1}^{j}$ if and only if $\left(x_{i}, x_{j}\right) \in \mathcal{E}_{A_{k}}$; the weight on such an edge is $w_{k}\left(x_{i}, x_{j}\right)$. Analogously, the edges between vertices $u_{k}^{i}$ and $x_{k+1}^{j}$, between $x_{k}^{i}$ and $y_{k}^{j}$, and between $u_{k}^{i}$ and $y_{k}^{j}$ are obtained with the corresponding edge weights. For a pictorial description of the aforesaid, see Figure 2.

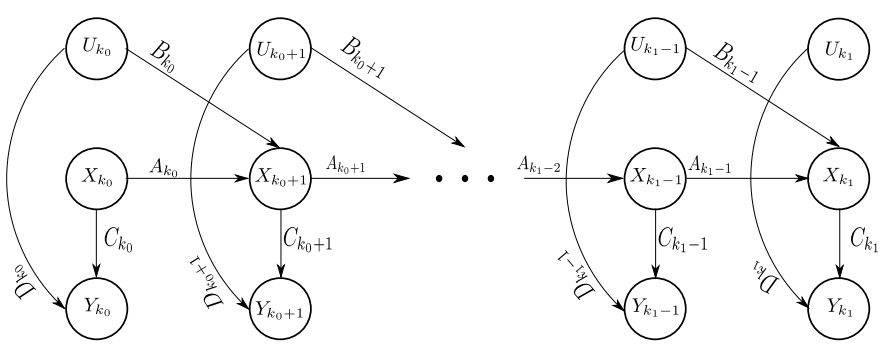

Fig. 2: Dynamic graph $\mathcal{S}_{k_{0}, k_{1}}$

Let $\bar{X}=X_{k_{0}} \cup X_{k_{0}+1} \cup \ldots \cup X_{k_{1}}$. Analogously, we define $\bar{U}$ and $\bar{Y}$. Notice that, for $\Psi_{k_{0}, k_{1}}$, the rows correspond to vertices in $\bar{Y}$, while the first $N$ columns correspond to vertices of $X_{k_{0}}$, and the remaining $\left(k_{1}-k_{0}+1\right) P$ columns correspond to vertices of $\bar{U}$.

In the dynamic graph $\mathcal{S}_{k_{0}, k_{1}}$ we define the cost of a path as the product of the weights along the edges of the path.

The following remark describes the relation between entries of matrix $\Psi_{k_{0}, k_{1}}$ and the cost of paths from $X_{k_{0}} \cup \bar{U}$ to $\bar{Y}$ in the dynamic graph.

Remark 1: Notice that $\left[C_{k+\ell} A_{k+\ell-1} \ldots A_{k}\right]_{i j}=$ $\sum_{h_{1}} \sum_{h_{2}} \ldots \sum_{h_{\ell}}\left[C_{k+\ell}\right]_{i h_{\ell}}\left[A_{k+\ell-1}\right]_{h_{\ell} h_{\ell-1}} \ldots\left[A_{k}\right]_{h_{0} j}$, and hence it equals the sum of costs over all paths from $x_{k}^{j}$ to $y_{k+\ell}^{i}$ in $\mathcal{S}_{k_{0}, k_{1}}$. Similarly, $\left[C_{k+\ell} A_{k+\ell-1} \ldots A_{k+1} B_{k}\right]_{i j}=$ $\sum_{h_{1}} \sum_{h_{2}} \ldots \sum_{h_{\ell}}\left[C_{k+\ell}\right]_{i h_{\ell}}\left[A_{k+\ell-1}\right]_{h_{\ell} h_{\ell-1}} \ldots\left[A_{k+1}\right]_{h_{1} h_{0}}\left[B_{k}\right]_{h_{0} j}$, and hence it equals the sum of costs over all paths from $u_{k}^{j}$ to $y_{k+\ell}^{i}$ in $\mathcal{S}_{k_{0}, k_{1}}$.

\section{E. Dynamic bipartite graph}

Similar to the dynamic graph $\mathcal{S}_{k_{0}, k_{1}}$ introduced in the previous subsection, there exists yet another equivalent graphical representation: the dynamic bipartite graph. In this subsection we shall acquaint ourselves with the same.

Notice that $\mathcal{J}_{k_{0}, k_{1}}$ is a structured matrix and let $\mathcal{B}\left(\mathcal{J}_{k_{0}, k_{1}}\right)$ be the bipartite graph associated with it, as described in Sect. IV-C. We shall refer to $\mathcal{B}\left(\mathcal{J}_{k_{0}, k_{1}}\right)$ as the dynamic bipartite graph of the system. In $\mathcal{B}\left(\mathcal{J}_{k_{0}, k_{1}}\right)$, the left vertex set (corresponding to columns of $\mathcal{J}_{k_{0}, k_{1}}$ ) is $\bar{U} \cup \bar{X}$. The right vertex set (corresponding to rows) is $\bar{X}^{\prime} \cup \bar{Y}$, where $\bar{X}^{\prime}=X_{k_{0}+1}^{\prime} \cup \ldots \cup X_{k_{1}}^{\prime}$, each $X_{k}^{\prime}$ denoting a copy of the set $X_{k}$. We will use the notation $x_{k}^{i \prime}$ for $i$ th vertex in the set $X_{k}^{\prime}$. In order to better highlight the connection between $\mathcal{B}\left(\mathcal{J}_{k_{0}, k_{1}}\right)$ and $\mathcal{S}_{k_{0}, k_{1}}$, it is convenient to draw $\mathcal{B}\left(\mathcal{J}_{k_{0}, k_{1}}\right)$ reordering the left vertex set as $X_{k_{0}}, U_{k_{0}}, X_{k_{0}+1}, U_{k_{0}+1}, \ldots, X_{k_{1}}, U_{k_{1}}$, and the right vertex set as $Y_{k_{0}}, X_{k_{0}+1}^{\prime}, Y_{k_{0}+1}, X_{k_{0}+2}^{\prime}, \ldots$, $Y_{k_{1}}$, as it is illustrated in Figure 3. Some non-zero entries of $\mathcal{J}_{k_{0}, k_{1}}$ are equal to -1 (within the $-I$ blocks); we will refer to the corresponding edges in $\mathcal{B}\left(\mathcal{J}_{k_{0}, k_{1}}\right)$ as new edges. All other non-zero entries of $\mathcal{J}_{k_{0}, k_{1}}$ are independent free parameters, obtained by looking at the matrices $\left\{A_{k}\right\}_{k_{0}}^{k_{1}-1}$, $\left\{B_{k}\right\}_{k_{0}}^{k_{1}-1},\left\{C_{k}\right\}_{k_{0}}^{k_{1}}$ and $\left\{D_{k}\right\}_{k_{0}}^{k_{1}}$; all such edges are in one-toone correspondence with the edges in $\mathcal{S}_{k_{0}, k_{1}}$, and we will call them old edges. 


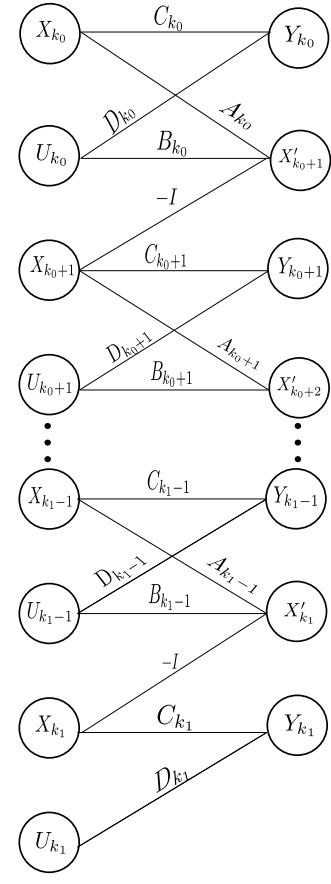

Fig. 3: Dynamic bipartite graph $\mathcal{B}\left(\mathcal{J}_{k_{0}, k_{1}}\right)$

\section{Structural ISO}

The main objective of this section is to seek graphical conditions such that the system (1) is ISO for almost all choices of edge weights in $\left\{\mathcal{G}_{k}\right\}$, where "almost all" means for all choices of free parameters except for those (possibly) lying on an algebraic variety of the space of free parameters [5]. Towards this end, we first define structural ISO and thereafter provide a graphical characterization for the same.

We define structural ISO as follows:

Definition 8: System (1) with the pattern of fixed zeros given by the graphs $\left\{\mathcal{G}_{k}\right\}$ is structurally ISO over $\left[k_{0}, k_{1}\right]$, if system (1) is ISO for almost all choices of edge weights in $\left\{\mathcal{G}_{k}\right\}$.

Recall that each choice of edge weight in $\left\{\mathcal{G}_{k}\right\}$ represents a choice of the free parameters of the structured system matrices $\left(A_{k}, B_{k}, C_{k}, D_{k}\right)$.

\section{A. Main Result}

Theorem 1: Consider the system (1) with the pattern of fixed zeros given by the graphs $\left\{\mathcal{G}_{k}\right\}$. Let $\mathcal{S}_{k_{0}, k_{1}}$ and $\mathcal{B}\left(\mathcal{J}_{k_{0}, k_{1}}\right)$ be the corresponding dynamic graph and dynamic bipartite graph, respectively. The following statements are equivalent:

(a) System (1) is structurally ISO over $\left[k_{0}, k_{1}\right]$;

(b) there exists a linking of size $N+\left(k_{1}-k_{0}\right) P+$ term-rank $\left(D_{k_{1}}\right)$ from $X_{k_{0}} \cup \bar{U}$ to $\bar{Y}$ in the dynamic graph $\mathcal{S}_{k_{0}, k_{1}}$

(c) there exists a matching of size $\left(k_{1}-k_{0}+1\right) N+\left(k_{1}-\right.$ $\left.k_{0}\right) P+\operatorname{term-rank}\left(D_{k_{1}}\right)$ in the dynamic bipartite graph $\mathcal{B}\left(\mathcal{J}_{k_{0}, k_{1}}\right)$.

Our main result should be understood in the following sense: if the condition is satisfied, then, over $\left[k_{0}, k_{1}\right]$, for almost all choices of free parameters except (possibly) for those lying on

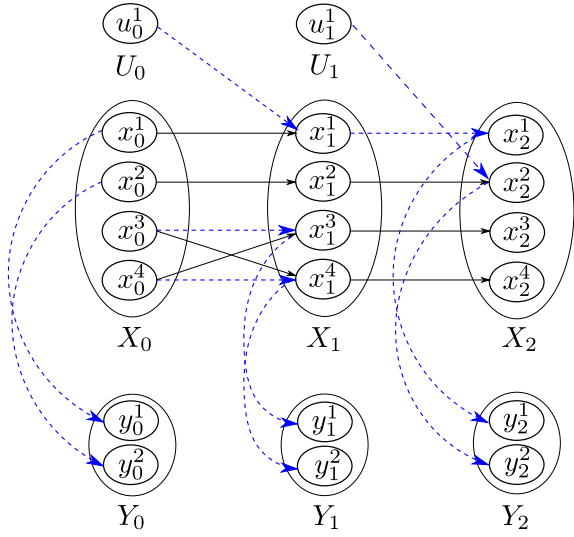

Fig. 4: Dynamic graph $\mathcal{S}_{0,2}$ for Example 1. The edges in dashed blue form a linking, $L_{0,2}$, from $X_{0} \cup \bar{U}$ to $\bar{Y}$ on $\mathcal{S}_{0,2}$

a subvariety of the space of free parameters, the corresponding system is ISO. On the other hand, if the condition is violated, then, over $\left[k_{0}, k_{1}\right]$, for almost all choices of free parameters, the corresponding system is not ISO. However, for at most few choices of free parameters, over $\left[k_{0}, k_{1}\right]$, the corresponding system might be ISO. We would like to point out that for the case of zero-feedthrough, if the given condition is violated, then since $D_{k_{1}}=0$, from Prop. 6 in [13], for every choice of free parameters, the corresponding system is not ISO.

We shall now apply the conditions given in Theorem 1 to the example given in Figure 1.

Example 1: Consider the time-varying system shown in Figure 1. Here $N=4, M=2, P=1$ and $D_{k}=0_{2 \times 1}$, for all $k$. We consider the time interval [0 2 [ ]. The dynamic graph $\mathcal{S}_{0,2}$ associated with it is given in Figure 4, wherein it is highlighted, in dashed blue, a collection of vertex-disjoint paths, namely $x_{0}^{1} \rightarrow y_{0}^{1}, x_{0}^{2} \rightarrow y_{0}^{2}, x_{0}^{3} \rightarrow x_{1}^{3} \rightarrow y_{1}^{1}, x_{0}^{4} \rightarrow$ $x_{1}^{4} \rightarrow y_{1}^{2}, u_{0}^{1} \rightarrow x_{1}^{1} \rightarrow x_{2}^{1} \rightarrow y_{2}^{1}, u_{1}^{1} \rightarrow x_{2}^{2} \rightarrow y_{2}^{2}$. Thus, condition (b) in Theorem 1 is satisfied, and consequently the structured system represented by Figure 1 is structurally ISO over the interval $[0,2]$.

\section{B. Proof of Theorem 1}

We prove the following circular implications: (b) implies (a), (a) implies (c), and (c) implies (b). Throughout this proof, we will use the short-hand notation $m_{1}=N+\left(k_{1}-k_{0}\right) P+$ term-rank $\left(D_{k_{1}}\right), m_{2}=\left(k_{1}-k_{0}+1\right) N+\left(k_{1}-k_{0}\right) P+$ term-rank $\left(D_{k_{1}}\right)$.

Proof of $(b) \Longrightarrow(a)$ : We divide this proof in two parts: first, given a linking as in (b) we construct one choice of free parameters such that this particular numerical realization of the system is ISO over $\left[k_{0}, k_{1}\right]$; second, we show that this further implies that the system is structurally ISO over $\left[k_{0}, k_{1}\right]$.

Part 1. By assumption, in the dynamic graph $\mathcal{S}_{k_{0}, k_{1}}$ there is a linking $L$ from $X_{k_{0}} \cup \bar{U}$ to $\bar{Y}$, of size $m_{1}$. We choose the free parameters according to the following construction. If there exists an edge in $L$ between $x_{k}^{j}$ and $x_{k+1}^{i}$, then set $\left[A_{k}\right]_{i j}$ to 1 else set it to 0 . Similarly, if there exists an edge in $L$ between $u_{k}^{j}$ and $x_{k+1}^{i}$, then set $\left[B_{k}\right]_{i j}$ to 1 else set it to 
0 . If there exists an edge in $L$ between $x_{k}^{j}$ and $y_{k}^{i}$, then set $\left[C_{k}\right]_{i j}$ to 1 else set it to 0 . Similarly, if there exists an edge in $L$ between $u_{k}^{j}$ and $y_{k}^{i}$, then set $\left[D_{k}\right]_{i j}$ to 1 else set it to 0 . Recall that Remark 1 relates entries of $\Psi_{k_{0}, k_{1}}$ and costs of paths in $\mathcal{S}_{k_{0}, k_{1}}$ : labeling columns of $\Psi_{k_{0}, k_{1}}$ with vertices in $X_{k_{0}} \cup \bar{U}$ and rows with vertices in $\bar{Y}$, the entry in row $y$ and column $v$ is equal to the sum of costs of all paths from $v$ to $y$. The key remark is that, with our choice of parameters, if a path in $\mathcal{S}_{k_{0}, k_{1}}$ belongs to the linking $L$, it has cost 1 ; otherwise cost 0 . This, together with the definition of linking, implies that $\Psi_{k_{0}, k_{1}}$ has $m_{1}$ columns (corresponding to vertices in $X_{k_{0}} \cup \bar{U}$ saturated by $L$ ) such that each column has exactly one 1 and all other entries are 0 , and moreover the 1's are in distinct rows (since paths in the linking are vertex-disjoint, their final vertices in $\bar{Y}$ are all distinct). The remaining $P$-term-rank $\left(D_{k_{1}}\right)$ columns are all-zero. Therefore, $\operatorname{rank}\left(\Psi_{k_{0}, k_{1}}\right)=m_{1}$.

Part 2. Given one choice of parameters such that $\operatorname{rank}\left(\Psi_{k_{0}, k_{1}}\right)=m_{1}$, we show that gen-rank $\left(\Psi_{k_{0}, k_{1}}\right)=m_{1}$, and hence the system is structurally ISO over $\left[k_{0}, k_{1}\right]$ (by Prop. 1).

By item 1) of Lemma 1, the generic rank is the maximum rank among all choices of parameters. Having one choice of parameters giving rank $m_{1}$, we have gen-rank $\left(\Psi_{k_{0}, k_{1}}\right) \geq m_{1}$. Then we notice that, for any choice of parameters, $\Psi_{k_{0}, k_{1}}$ can have at most $m_{1}$ independent columns, since the rank of the block with the last $P$ columns is rank $D_{k_{1}} \leq$ term-rank $D_{k_{1}}$. This shows that gen-rank $\left(\Psi_{k_{0}, k_{1}}\right) \leq m_{1}$, ending the proof.

Proof of $(a) \Longrightarrow(c)$ : We prove that negating (c) implies negating (a).

Suppose that there exists no matching of size $m_{2}$ in the dynamic bipartite graph $\mathcal{B}\left(\mathcal{J}_{k_{0}, k_{1}}\right)$, which implies term-rank $\left(\mathcal{J}_{k_{0}, k_{1}}\right)<m_{2}$. This implies that, for all parameter choices, $\operatorname{rank}\left(\mathcal{J}_{k_{0}, k_{1}}\right)<m_{2}$; indeed, by items 1) and 3) in Lemma 1, $\operatorname{rank}\left(\mathcal{J}_{k_{0}, k_{1}}\right) \leq \operatorname{term-rank}\left(\mathcal{J}_{k_{0}, k_{1}}\right)$. Moreover, since $D_{k_{1}}$ is a pattern matrix, by item 3) in Lemma 1 $\operatorname{gen-rank}\left(D_{k_{1}}\right)=\operatorname{term} \operatorname{rank}\left(D_{k_{1}}\right)$, i.e., $\operatorname{rank}\left(D_{k_{1}}\right)=$ term-rank $\left(D_{k_{1}}\right)$ for almost all parameters. Hence, except for the parameters belonging to the proper subvariety where $\operatorname{rank}\left(D_{k_{1}}\right)<\operatorname{term}-\operatorname{rank}\left(D_{k_{1}}\right), \operatorname{rank}\left(\mathcal{J}_{k_{0}, k_{1}}\right)<m_{2}$. Therefore, from Prop. 2, system (1) is not structurally ISO over $\left[k_{0}, k_{1}\right]$.

Proof of $(c) \Longrightarrow(b)$ : Given a matching as in (c), we construct a linking $L$ as in (b).

Consider a given matching $\mathcal{M}$ in $\mathcal{B}\left(\mathcal{J}_{k_{0}, k_{1}}\right)$, of size $m_{2}$. The first remark is that $\mathcal{M}$ saturates all vertices of $\bar{X} \cup \bar{U} \backslash U_{k_{1}}$, and exactly term-rank $\left(D_{k_{1}}\right)$ vertices of $U_{k_{1}}$. Indeed, any matching in $\mathcal{B}\left(\mathcal{J}_{k_{0}, k_{1}}\right)$ can saturate at most term-rank $\left(D_{k_{1}}\right)$ vertices of $U_{k_{1}}$ (since vertices in $U_{k_{1}}$ only have edges corresponding to $\left.D_{k_{1}}\right)$, and at most $\left|\bar{X} \cup \bar{U} \backslash U_{k_{1}}\right|=m_{2}-\operatorname{term}-\operatorname{rank}\left(D_{k_{1}}\right)$ vertices of $\bar{X} \cup \bar{U} \backslash U_{k_{1}}$, so that $\mathcal{M}$ must achieve both bounds with equality.

Recall that edges of $\mathcal{B}\left(\mathcal{J}_{k_{0}, k_{1}}\right)$ are partitioned in old edges, in one-to-one correspondence with edges in $\mathcal{S}_{k_{0}, k_{1}}$, and new edges of the form $\left(x_{k}^{j}, x_{k}^{j \prime}\right)$. We will show how to find a suitable set of old edges from $\mathcal{M}$, such that the corresponding edges in $\mathcal{S}_{k_{0}, k_{1}}$ form a linking $L$ with $m_{1}$ paths; such paths will start from a) all the vertices of $X_{k_{0}}$, and b) those vertices of $\bar{U}$ saturated by $\mathcal{M}$, and will end in $\bar{Y}$. The construction is the following. Starting from $x_{k_{0}}^{j} \in X_{k_{0}}$, we consider the edge that saturates $x_{k_{0}}^{j}$ in $\mathcal{M}$. If this edge is $\left(x_{k_{0}}^{j}, y_{k_{0}}^{i}\right)$ for some $y_{k_{0}}^{i} \in \bar{Y}$, we add $\left(x_{k_{0}}^{j}, y_{k_{0}}^{i}\right)$ as a path of length 1 in $L$. Else, the edge is $\left(x_{k_{0}}^{j}, x_{k_{0}+1}^{j_{1}{ }^{\prime}}\right)$ for some $x_{k_{0}+1}^{j_{1}{ }^{\prime}} \in \bar{X}^{\prime}$. In the latter case, we add the edge $\left(x_{k_{0}}^{j}, x_{k_{0}+1}^{j_{1}}\right)$ to our path construction and then we look at the vertex $x_{k_{0}+1}^{j_{1}}$ in $\mathcal{B}\left(\mathcal{J}_{k_{0}, k_{1}}\right)$, noticing that it is saturated in $\mathcal{M}$ by some old edge. Indeed, it cannot be saturated by its new edge $\left(x_{k_{0}+1}^{j_{1}}, x_{k_{0}+1}^{j_{1} \prime}\right)$, since $x_{k_{0}+1}^{j_{1} \prime}$ is already saturated by $\left(x_{k_{0}}^{j}, x_{k_{0}+1}^{j_{1} \prime}\right)$. Consequently, $x_{k_{0}+1}^{j_{1}+1}$ is saturated by an old edge, connecting it either to $y_{k_{0}+1}^{i}$ or to $x_{k_{0}+2}^{j_{2} \prime}$, and we can include the corresponding edge in our path construction. The process repeats until a vertex in $\bar{Y}$ is reached. Notice that this process surely reached $\bar{Y}$. Indeed, recall that all vertices in $\bar{X}$ are saturated; moreover, in case the construction does not reach any vertex in $\bar{Y} \backslash Y_{k_{1}}$, then it reaches some vertex in $X_{k_{1}}$, and from there, it necessarily reaches a vertex in $Y_{k_{1}}$, because there is no vertex set $X_{k_{1}+1}$.

This construction gives paths from each vertex of $X_{k_{0}}$ to $\bar{Y}$ in $\mathcal{S}_{k_{0}, k_{1}}$. Analogously, paths can be constructed from every $u_{k}^{j} \in \bar{U} \backslash U_{k_{1}}$ and for every $u_{k_{1}}^{j} \in U_{k_{1}}$ saturated by $\mathcal{M}$.

By construction, since all edges in the paths correspond to some edges in $\mathcal{M}$, the paths are vertex-disjoint. Hence, by Definition 3, they form a linking from $X_{k_{0}} \cup \bar{U}$ to $\bar{Y}$ in the dynamic graph $\mathcal{S}_{k_{0}, k_{1}}$. Moreover, the linking has size $m_{1}$, thus ending the proof.

An illustration of this proof technique is as follows: $\mathcal{B}\left(\mathcal{J}_{0,2}\right)$ is the dynamic bipartite graph shown in Figure 5. A matching $\mathcal{M}_{0,2}$, on $\mathcal{B}\left(\mathcal{J}_{0,2}\right)$, that saturates all the vertices in $X_{0} \cup U_{0} \cup$ $X_{1} \cup U_{1} \cup X_{2}$ is shown in Figure 6a. Notice that in Figure 6a the matching $\mathcal{M}_{0,2}$ connects vertices in $X_{0} \cup \bar{U}$ to $\bar{Y}$ in the following manner: $x_{0}^{1} \rightarrow y_{0}^{1}, x_{0}^{2} \rightarrow y_{0}^{2}, x_{0}^{3} \rightarrow x_{1}^{3 \prime} x_{1}^{3} \rightarrow y_{1}^{1}$, $x_{0}^{4} \rightarrow x_{1}^{4^{\prime}} x_{1}^{4} \rightarrow y_{1}^{2}, u_{0}^{1} \rightarrow x_{1}^{1 \prime} x_{1}^{1} \rightarrow x_{1}^{2 \prime} x_{1}^{2} \rightarrow y_{2}^{1}, u_{1}^{1} \rightarrow x_{2}^{2 \prime}$ $x_{2}^{2} \rightarrow y_{2}^{2}$. Hence, we can construct a linking of size 6 , on $\mathcal{S}_{0,2}$, from $X_{0} \cup \bar{U}$ to $\bar{Y}$, as depicted in Figure 4 .

\section{S-STRUCTURAL ISO}

The main objective of this section is to seek graphical conditions such that the system (1) is ISO for all non-zero edge weights in $\left\{\mathcal{G}_{k}\right\}$. Notice that, under such a setting, each and every element in a vector of free parameters should be nonzero. Towards this end, we first define s-structural ISO and thereafter provide a graphical characterization for the same.

Definition 9: System (1) with the pattern of fixed zeros given by the graphs $\left\{\mathcal{G}_{k}\right\}$ is s-structurally ISO over $\left[k_{0}, k_{1}\right]$, if system (1) is ISO for all non-zero choices of edge weights in $\left\{\mathcal{G}_{k}\right\}$.

Definition 9 should be understood as follows: System (1) being s-structurally ISO means that for every non-zero choice of edge weights in $\left\{\mathcal{G}_{k}\right\}$ (or equivalently, for every non-zero choice of free parameters in the structured system matrices), the corresponding quadruplet $\left\{A_{k}, B_{k}, C_{k}, D_{k}\right\}$ satisfies $\operatorname{rank}\left(\mathcal{J}_{k_{0}, k_{1}}\right)=\left(k_{1}-k_{0}+1\right) N+\left(k_{1}-k_{0}\right) P+\operatorname{rank}\left(D_{k_{1}}\right)$. 


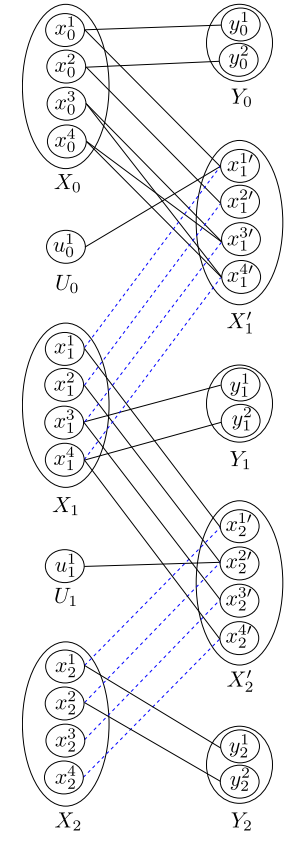

Fig. 5: Dynamic bipartite graph $\mathcal{B}\left(\mathcal{J}_{0,2}\right)$ of Example 1. The edges in black are old edges, in one-to-one correspondence with the edges in the dynamic graph $\mathcal{S}_{0,2}$ shown in Figure 4, while the edges in dashed blue are new edges, corresponding to blocks $-I_{4 \times 4}$ in $\mathcal{J}_{0,2}$

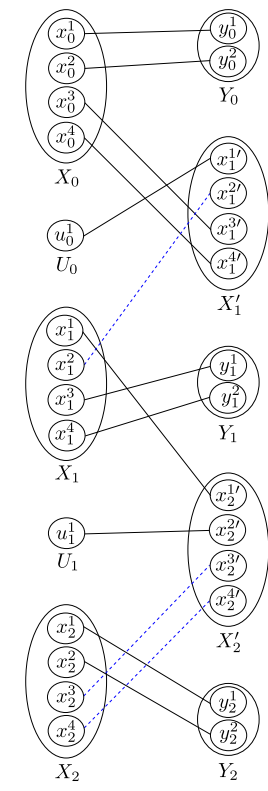

(a) $\mathcal{M}_{0,2}$

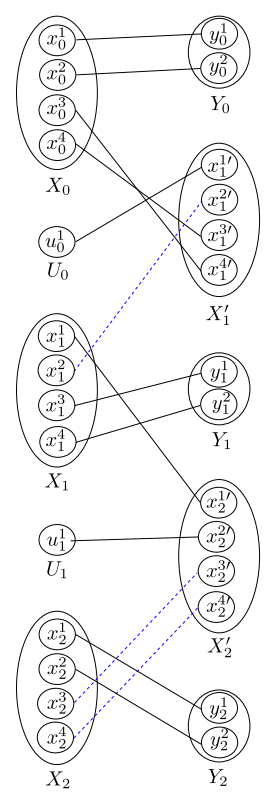

(b) $\tilde{\mathcal{M}}_{0,2}$
Fig. 6: Matchings on the dynamic bipartite graph $\mathcal{B}\left(\mathcal{J}_{0,2}\right)$ of Example 1

\section{A. Main Result}

It turns out that thanks to Definition 5 and Lemma 2 one can obtain a sufficient condition for s-structural ISO. In order to find a necessary condition for s-structural ISO, we restrict our attention to the following submatrix of $\mathcal{J}_{k_{0}, k_{1}}$ :

$$
\mathcal{P}_{k_{0}, k_{1}}=\left[\begin{array}{cc}
\mathbf{D}_{k_{0}, k_{1}-1} & \\
0_{M \times\left(k_{1}-k_{0}\right) P} & \mathcal{Q}_{k_{0}, k_{1}} \\
\mathbf{B}_{k_{0}, k_{1}-1} &
\end{array}\right]
$$

where $\mathcal{P}_{k_{0}, k_{1}}$ has $\left(k_{1}-k_{0}+1\right) M+\left(k_{1}-k_{0}\right) N$ rows and $\left(k_{1}-k_{0}\right) P+\left(k_{1}-k_{0}+1\right) N$ columns, and let $\mathcal{B}\left(\mathcal{P}_{k_{0}, k_{1}}\right) \subseteq$ $\mathcal{B}\left(\mathcal{J}_{k_{0}, k_{1}}\right)$ be the bipartite graph associated with $\mathcal{P}_{k_{0}, k_{1}}$. With these in place, our second main result is stated as follows:

Theorem 2: Consider the system (1) with the pattern of fixed zeros given by the graphs $\left\{\mathcal{G}_{k}\right\}$. Let $\mathcal{B}\left(\mathcal{J}_{k_{0}, k_{1}}\right)$ and $\mathcal{B}\left(\mathcal{P}_{k_{0}, k_{1}}\right)$ be as defined. System (1) is s-structurally ISO, over $\left[k_{0}, k_{1}\right]$,

1) if there exists a uniquely restricted matching of size $\left(k_{1}-\right.$ $\left.k_{0}+1\right) N+\left(k_{1}-k_{0}\right) P+\operatorname{term}-\operatorname{rank}\left(D_{k_{1}}\right)$ in $\mathcal{B}\left(\mathcal{J}_{k_{0}, k_{1}}\right)$.

2) only if there exists a uniquely restricted matching of size

$$
\left(k_{1}-k_{0}\right) P+\left(k_{1}-k_{0}+1\right) N \text { in } \mathcal{B}\left(\mathcal{P}_{k_{0}, k_{1}}\right) \text {. }
$$

Notice that there exists a gap between the sufficient condition and the necessary conditions given in Theorem 2, as the latter pertains to a submatrix, namely $\mathcal{P}_{k_{0}, k_{1}}$. However, for the case when $D_{k_{1}}=0$, from Theorem 2, we can obtain a full characterization of s-structural ISO, and is given as follows.

Corollary 1: If $D_{k_{1}}=0$, then system (1) is s-structurally ISO, over $\left[k_{0}, k_{1}\right]$, if and only if there exists a uniquely restricted matching of size $\left(k_{1}-k_{0}\right) P+\left(k_{1}-k_{0}+1\right) N$ in $\mathcal{B}\left(\mathcal{J}_{k_{0}, k_{1}}\right)$.

We shall now apply the conditions given in Theorem 2 to our example shown in Figure 1.

Example 1 (continued): Notice that matching $\mathcal{M}_{0,2}$, see Figure $6 \mathrm{a}$, in $\mathcal{B}\left(\mathcal{J}_{0,2}\right)$ saturates all the vertices in $X_{0} \cup U_{0} \cup X_{1} \cup$ $U_{1} \cup X_{2}$. It can also be seen that over the same choice of vertex sets, there exists another matching, namely $\tilde{\mathcal{M}}_{0,2}$, as depicted in Figure 6b. Moreover, there does not exist another choice of vertex sets of size 14 either in the left vertex set, i.e., $X_{0} \cup U_{0} \cup X_{1} \cup U_{1} \cup X_{2}$, or in the right vertex set, i.e., $Y_{0} \cup X_{1}^{\prime} \cup Y_{1} \cup X_{2}^{\prime} \cup Y_{2}$. Consequently, there does not exist a uniquely restricted matching of size 14 in $\mathcal{B}\left(\mathcal{J}_{0,2}\right)$. Therefore, from item 2) in Theorem 2, we can conclude that the structured system shown in Figure 1 is not s-structurally ISO.

\section{B. Proof of Theorem 2}

First we prove that the condition given in item 1) is sufficient for s-structural ISO over $\left[k_{0}, k_{1}\right]$, and then we prove that the condition given in item 1 ) is necessary for s-structural ISO over $\left[k_{0}, k_{1}\right]$.

Proof of sufficiency of 1): Recall that some non-zero entries of $\mathcal{J}_{k_{0}, k_{1}}$ are -1 , and all other non-zero entries are distinct free parameters. Let $\overline{\mathcal{J}}_{k_{0}, k_{1}}$ be the pattern matrix equal to $\mathcal{J}_{k_{0}, k_{1}}$, except that the -1 's are replaced by free parameters, distinct from each other and from other parameters, so that $\overline{\mathcal{J}}_{k_{0}, k_{1}}$ is a pattern matrix, and $\mathcal{J}_{k_{0}, k_{1}}$ is obtained from it by fixing some parameters to -1 .

The assumption in 1) is that there exists a uniquely restricted matching of size $\left(k_{1}-k_{0}+1\right) N+\left(k_{1}-k_{0}\right) P+$ $\operatorname{term}-\operatorname{rank}\left(D_{k_{1}}\right)$ in the dynamic bipartite graph $\mathcal{B}\left(\mathcal{J}_{k_{0}, k_{1}}\right)$. Notice that $\mathcal{B}\left(\mathcal{J}_{k_{0}, k_{1}}\right)=\mathcal{B}\left(\overline{\mathcal{J}}_{k_{0}, k_{1}}\right)$. Therefore, from Lemma 2 , the matrix $\mathcal{J}_{k_{0}, k_{1}}$ has rank equal to $\left(k_{1}-k_{0}+1\right) N+$ $\left(k_{1}-k_{0}\right) P+\operatorname{term}-\operatorname{rank}\left(D_{k_{1}}\right)$ for all non-zero choices of 
free parameters. In particular, this remains true when fixing some parameters to -1 to obtain $\mathcal{J}_{k_{0}, k_{1}}$. Therefore, from Definition 9, System (1) is s-structurally ISO over $\left[k_{0}, k_{1}\right]$.

Proof of necessity of 2): In order to prove that the condition in 2) is necessary, we prove a slightly stronger result, given in Lemma 3 below, involving submatrices of $\mathcal{P}_{k_{0}, k_{1}}$ obtained considering $r$ columns, and involving the corresponding bipartite graph as described in Sect. IV-C.

Lemma 3: Let $r$ be any integer $1 \leq r \leq\left(k_{1}-k_{0}\right) P+\left(k_{1}-\right.$ $\left.k_{0}+1\right) N$. For any submatrix $\mathcal{L}$ formed with $r$ columns of $\mathcal{P}_{k_{0}, k_{1}}$, if there exists no uniquely restricted matching of size $r$ in the associated bipartite graph $\mathcal{B}(\mathcal{L})$, then there exists a non-zero choice of free parameters such that the corresponding numerical realization of $\mathcal{L}$ has $\operatorname{rank} \mathcal{L}<r$.

The Lemma's statement specialized to $r=\left(k_{1}-k_{0}\right) P+$ $\left(k_{1}-k_{0}+1\right) N$ concerns $\mathcal{L}=\mathcal{P}_{k_{0}, k_{1}}$ and gives the desired result: if there exists no uniquely restricted matching of size $\left(k_{1}-k_{0}\right) P+\left(k_{1}-k_{0}+1\right) N$ in the dynamic bipartite graph $\mathcal{B}\left(\mathcal{P}_{k_{0}, k_{1}}\right)$, then there exists a non-zero choice of free parameters such that the corresponding numerical realization of $\mathcal{P}_{k_{0}, k_{1}}$ has rank $\mathcal{P}_{k_{0}, k_{1}}<\left(k_{1}-k_{0}\right) P+\left(k_{1}-k_{0}+1\right) N$; this further implies that there is a non-zero choice of parameters for which $\operatorname{rank} \mathcal{J}_{k_{0}, k_{1}}<\left(k_{1}-k_{0}\right) P+\left(k_{1}-k_{0}+1\right) N+$ term-rank $\left(D_{k_{1}}\right)$, and hence the system is not s-structurally ISO over $\left[k_{1}, k_{1}\right]$.

The formulation for general $r$ is introduced because it allows a proof by induction on $r$, as follows.

Proof of Lemma 3: The proof is by induction on $r$, similar to the proof of Theorem 3.4 in [19], but adapted to the fact that $\mathcal{P}_{k_{0}, k_{1}}$ is not a pattern matrix, since it has some entries fixed to -1 . It will be crucial to recall that $\mathcal{P}_{k_{0}, k_{1}}$ inherits from $\mathcal{J}_{k_{0}, k_{1}}$ the following property: its entries are some 0 's, some distinct free parameters, and some -1 's, with at most one -1 per row.

The base case is $r=1$, where $\mathcal{L}$ has only one column, and $\mathcal{B}(\mathcal{L})$ has only one vertex in its left vertex set. A matching of size 1 is simply one edge; by Definition 5, every matching of size 1 is a uniquely restricted matching. Consequently, if there exist no uniquely restricted matching of size 1 in $\mathcal{B}(\mathcal{L})$, then there exists no matching in $\mathcal{B}(\mathcal{L})$, and hence there exists no edge either. This implies that $\mathcal{L}$ is all-zero, so that $\operatorname{rank}(\mathcal{L})=$ 0 .

Now we assume that the claim holds for $r-1$ (inductive assumption), and we prove that this implies the claim holds for $r$. We consider any $\mathcal{L}$ obtained taking $r$ columns of $\mathcal{P}_{k_{0}, k_{1}}$. Matrix $\mathcal{L}$ might or might not have a row with exactly one nonzero element, and the two cases require a different proof.

Case a: there exists a row $i$ of $\mathcal{L}$ having exactly one nonzero term; say this term is in position $(i, j)$.

Let $\mathcal{L}_{:,-j}$ denote the submatrix of $\mathcal{L}$ obtained by removing the $j$ th column; notice that this is a matrix containing some $r-1$ columns of $\mathcal{P}_{k_{0}, k_{1}}$. By assumption, there is no uniquely restricted matching of size $r$ in $\mathcal{B}(\mathcal{L})$. This implies that there is no uniquely restricted matching of size $r-1$ in $\mathcal{B}\left(\mathcal{L}_{:,-j}\right)$. Indeed, if there was one, then it would be possible to construct a uniquely restricted matching of size $r$ in $\mathcal{B}(\mathcal{L})$ by adding the edge corresponding to the $(i, j)$ th entry of $\mathcal{L}$.

Now we can apply the inductive assumption to $\mathcal{L}_{:,-j}$ : since it is a matrix formed with $r-1$ columns of $\mathcal{P}_{k_{0}, k_{1}}$, and having no uniquely restricted matching of size $r-1$ in the corresponding bipartite graph, there exists a non-zero choice of parameters such that the corresponding numerical realization of $\mathcal{L}_{:,-j}$ has $\operatorname{rank} \mathcal{L}_{:,-j}<r-1$. Then take the same parameters for the entries of $\mathcal{L}$, together with an arbitrary value for the $(i, j)$ th entry in case it is not already fixed to -1 ; this numerical realization of $\mathcal{L}$ has $\operatorname{rank} \mathcal{L}<r$.

Case b: no row of $\mathcal{L}$ has exactly one non-zero term. In this case we can find a non-zero choice of free parameters such that the corresponding numerical realization of $\mathcal{L}$ has $\operatorname{rank} \mathcal{L}<r$. without using the inductive assumption nor the assumption about non-existence of uniquely restricted matching. Indeed, we can find a non-zero choice of parameters such that all rows have zero row-sum, for example with the following construction. Recall that we are in case b) (no row has exactly one non-zero term) and that $\mathcal{L}$ is a submatrix of $\mathcal{P}_{k_{0}, k_{1}}$ and hence it has at most one -1 per row. Hence, there can be only three kinds of row: i) an all-zero row; ii) a row with exactly one -1 and with $p \geq 1$ free parameters; iii) a row with no -1 and with $p \geq 2$ free parameters. Rows of kind i) already have zero row-sum. For rows of kind ii), fix $p-1$ parameters to -1 (skipping this step if $p=1$ ), and then fix the last parameter to $p$. For rows of kind iii), fix $p-1$ parameters to -1 and then fix the last parameter to $p-1$. With this non-zero choice of parameters, the sum of elements along each row of $\mathcal{L}$ is 0 , which means that the sum of all columns of $\mathcal{L}$ is a zero vector and hence the columns are not linearly independent, so that this numerical realization of $\mathcal{L}$ has $\operatorname{rank} \mathcal{L}<r$.

\section{CONCLUSION}

This paper focuses on discrete-time linear network systems with time-varying topologies. Firstly, it gives a full characterization of structural ISO in terms of existence of a linking of an appropriate size on the corresponding dynamic graph. Secondly, for s-structural ISO, a sufficient condition and a necessary condition are provided. Furthermore, under suitable assumptions on the feedthrough matrix, namely $D_{k_{1}}=0$, a full characterization for s-structural ISO is also provided. We would like to point out that, for s-structural ISO, for the case of $D_{k_{1}} \neq 0$, there is a gap between necessary and sufficient conditions, and remains open. Also, throughout this paper we have assumed first order dynamics on each node. On the other hand, if the nodes were to have higher-order dynamics, then the local dynamics could impose constrains on the free parameters. This differs from the setting discussed in the present paper, and remains open.

\section{REFERENCES}

[1] C.-T. Lin, "Structural controllability," IEEE Transactions on Automatic Control, vol. 19, no. 3, pp. 201-208, 1974.

[2] R. W. Shields and J. B. Pearson, "Structural controllability of multi-input linear systems," Rice University ECE Technical Report, no. TR7502, 1975.

[3] H. Mayeda and T. Yamada, "Strong structural controllability," SIAM Journal on Control and Optimization, vol. 17, no. 1, pp. 123-138, 1979.

[4] K. Murota, Matrices and matroids for systems analysis. Springer Science \& Business Media, 2009, vol. 20. 
[5] J.-M. Dion, C. Commault, and J. Van Der Woude, "Generic properties and control of linear structured systems: a survey," Automatica, vol. 39, no. 7, pp. 1125-1144, 2003.

[6] P. Holme, "Network reachability of real-world contact sequences," Physical Review E, vol. 71, no. 4, p. 046119, 2005.

[7] T. Li and J.-F. Zhang, "Consensus conditions of multi-agent systems with time-varying topologies and stochastic communication noises," IEEE Transactions on Automatic Control, vol. 55, no. 9, pp. 2043-2057, 2010.

[8] H. J. Palanthandalam-Madapusi and D. S. Bernstein, "Unbiased minimum-variance filtering for input reconstruction," in American Control Conference, 2007. ACC'07. IEEE, 2007, pp. 5712-5717.

[9] S. Z. Yong, M. Zhu, and E. Frazzoli, "Switching and data injection attacks on stochastic cyber-physical systems: Modeling, resilient estimation and attack mitigation," arXiv preprint arXiv:1707.07112, 2017.

[10] F. Pasqualetti, A. Bicchi, and F. Bullo, "Consensus computation in unreliable networks: A system theoretic approach," IEEE Transactions on Automatic Control, vol. 57, no. 1, pp. 90-104, 2012.

[11] T. Boukhobza, F. Hamelin, and S. Martinez-Martinez, "State and input observability for structured linear systems: A graph-theoretic approach," Automatica, vol. 43, no. 7, pp. 1204-1210, 2007.

[12] F. Garin, "Structural Delay-1 Input-and-State Observability," in 56th IEEE Conference on Decision and Control, CDC 2017, no. 4, Melbourne, Australia, 2017, pp. 2324 - 2329.

[13] S. Gracy, F. Garin, and A. Y. Kibangou, "Structural and strong structural input and state observability of linear network systems," IEEE Transactions on Control of Network Systems, Appeared Online.

[14] A. Y. Kibangou, F. Garin, and S. Gracy, "Input and state observability of network systems with a single unknown input," ser. Proc. 6th IFAC Workshop on Distributed Estimation and Control in Networked Systems (NecSys). Tokyo, Japan: IFAC, Sep. 2016.

[15] S. Poljak, "On the generic dimension of controllable subspaces," IEEE Transactions on Automatic Control, vol. 35, no. 3, pp. 367-369, 1990.

[16] G. Reissig, C. Hartung, and F. Svaricek, "Strong structural controllability and observability of linear time-varying systems," IEEE Transactions on Automatic Control, vol. 59, no. 11, pp. 3087-3092, 2014.

[17] X. Liu, H. Lin, and B. M. Chen, "Structural controllability of switched linear systems," Automatica, vol. 49, no. 12, pp. 3531-3537, 2013.

[18] J. Massey and M. K. Sain, "Inverses of linear sequential circuits," IEEE Transactions on Computers, vol. 100, no. 4, pp. 330-337, 1968.

[19] D. Hershkowitz and H. Schneider, "Ranks of zero patterns and sign patterns," Linear and Multilinear Algebra, vol. 34, no. 1, pp. 3-19, 1993.

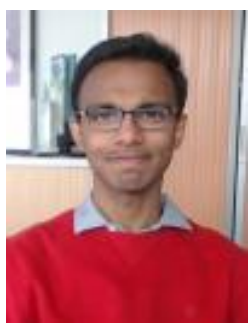

Sebin Gracy is a $\mathrm{PhD}$ student with $\mathrm{NeCS}$ team at INRIA and GIPSA-lab, Grenoble, France, since October, 2015. Prior to that, he obtained his MS and B.E. degrees in Electrical Engineering from the University of Colorado at Boulder and the University of Mumbai, in December, 2013 and June 2010, respectively.

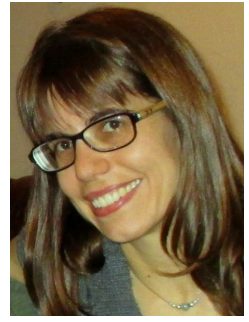

Federica Garin ederica Garinederica GarinF (M'16) is a researcher ('chargée de recherche') with the $\mathrm{NeCS}$ team at INRIA and GIPSA-lab, Grenoble (France). She received her B.S., M.S., and Ph.D. degrees in Applied Mathematics from Politecnico di Torino (Italy) in 2002, 2004, and 2008, respectively. She was a post-doctoral researcher at Universit di Padova (Italy) in 2008 and 2009, and at INRIA in 2010. She is an Associate Editor in the IEEE-CSS Conference Editorial Board and in the European Control Association (EUCA) Conference Editorial Board. Her current research interests are in distributed algorithms and network systems.

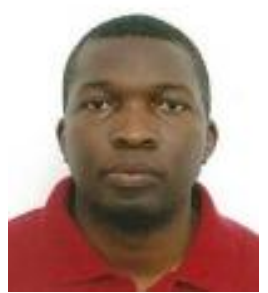

Alain Y. Kibangou (M'10) received the Ph.D. degree in control, signal, and image processing jointly from the University of Nice Sophia Antipolis, France, and from the Cadi Ayyad University of Marrakesh, Morocco, in 2005.

He was a Postdoctoral Researcher successively with the I3S Laboratory, Sophia Antipolis; the LAAS, Toulouse, France; and the GIPSA-Lab, Grenoble, France. In 2009, he became an Associate Professor within the framework of the "CNRSHigher Education chairs" program. Currently, he is an Associate Professor with the University of Grenoble Alpes and a Researcher with GipsaLab. His research interests include distributed estimation, wireless sensor networks, traffic modeling and prediction, filtering, identification, and tensor analysis. 